\title{
Higher Perceived Life Control Decreases Genetic Variance in Physical Health: Evidence From a National Twin Study
}

\author{
Wendy Johnson and Robert F. Krueger \\ University of Minnesota-Twin Cities
}

\begin{abstract}
Physical health has been linked consistently with both income and sense of control, and the authors previously demonstrated that genetic variation in physical health measures decreased with increasing income (see W. Johnson \& R. F. Krueger, 2004). Using a nationwide sample of 719 twin pairs from the MacArthur Foundation National Survey of Midlife Development in the United States, in this study the authors show that genetic variation in physical health measures (number of chronic illnesses and body mass index) also decreases with increasing sense of control. The authors integrate findings for income and control by demonstrating an interaction between genetic influences on sense of control and income in explaining physical health. They hypothesize that the mechanism underlying the interaction is the known biological relationship between metabolic efficiency and adaptation to stressful environments.
\end{abstract}

Physical health has consistently been linked with income, wealthier people being healthier. The relationship holds over time, in a variety of geographic settings, and for almost every disease and condition (Adler et al., 1994). The association is monotonic across the full range of income; it is not limited to a comparison of those with incomes below and above poverty levels. The relationship cannot be explained by lack of access to medical care, and it persists even in populations with universal health insurance (Adler $\&$ Snibbe, 2003). These findings suggest that the mechanisms underlying the relationship are more complex than previously believed. Simple differences in basic living conditions are unlikely to account for differences in health status between moderate and high levels of income.

Recent studies of mechanisms that may account for the relationship have focused on the hypothesis that income influences biological functions that in turn affect health status. The process, it is suspected, begins with increased physical and social demands and decreased resources for dealing with those demands associated with lower levels of income (Adler \& Snibbe, 2003). The result is greater psychological response to the stress generated by the mismatch between demands and resources, with subsequent physiological sequelae. Over time, the accumulation of such sequelae contributes to biological disregulation (McEwen, 1998), measured as allostatic load, which precipitates disease states. Allostatic load is considered to be a summary index of bodily wear and tear and includes systolic and diastolic blood pressure, waist-to-hip ratio, high- and low-density lipoprotein cholesterol, blood glycosylated hemoglobin (an indicator of blood glucose levels over the past 2-3

Wendy Johnson and Robert F. Krueger, Department of Psychology, University of Minnesota-Twin Cities.

This research was supported by the John D. and Catherine T. MacArthur Foundation Research Network on Successful Midlife Development and by National Institute on Aging Grant \#AG20166.

Correspondence concerning this article should be addressed to Wendy Johnson, Department of Psychology, University of Minnesota-Twin Cities, 75 East River Road, Minneapolis, MN 55455. E-mail: john4350@tc.umn.edu months), and the hormones dihydroepiandrosterone, epinephrine, and norepinephrine (indicators of malfunction of the fight-or-flight cortisol response system). These indicators are generally acknowledged to be under genetic influence (e.g., Komaroff, 1999), and this is also true of many of the most common chronically debilitating physical health conditions, including heart disease, arthritis, many cancers, and diabetes.

We recently demonstrated that genetic variance associated with each of two measures of physical health declined significantly with increasing income in a nationwide U.S. twin sample (Kessler, Gilman, Thornton, \& Kendler, 2004). The measures used were number of chronic illnesses and body mass index (BMI; Johnson $\&$ Krueger, 2004). The decline in variance with increasing income implies the existence of a gene-environment interaction, because the extent of genetic influence depends on individuals' environments. The interaction effect could not be removed by correcting income for the presence of health insurance coverage or for level of education. This finding suggests that some aspect of the objective individual environment created by income moderates gene expression related to physical disease conditions, and this effect is not related in an obvious way to access to health care or general education. We still do not know, however, how income may act to compress the genetic variance associated with physical health across the full range of income. That is, we do not know what it is about income that results in changes in genetic variation associated with physiological conditions.

Whatever it is, the effect appears to transcend specific disease categories, hinting at a very general influence, perhaps a personality variable. One personality variable likely involved is sense of control, which has consistently been linked with physical health (Rodin, 1986). Those who report a subjective sense that they have greater control over life outcomes report better health, fewer and more minor symptoms, faster recovery from illness, and reduced mortality (Rodin, Timko, \& Harris, 1985). Lachman and Weaver (1998) reported that this association holds within income levels. They also observed that individuals in the lowest income group with a high sense of control showed levels of physical health comparable with those in the higher income groups overall. In 
addition, Bailis, Segall, Mahon, Chipperfield, and Dunn (2001) found that although perceived control was systematically related to socioeconomic differences in self-rated health status, this was not the direct result of greater participation in health-related behaviors on the part of those with higher perceived control. Thus, there is evidence that control over life may be an aspect of personality that directly explains at least part of the income-health gradient.

One possible biological mechanism underlying the incomehealth gradient is suggested by data from research on humans and experimental animals indicating that genes for metabolic efficiency enable adaptation to stressful environments and play a primary role in affecting life span. By implication, these processes also influence health (Parsons, 2003). In Drosophila melanogaster, genetic variability for fitness, and especially for mortality, increases in situations of high stress (Parsons, 2002). Though it is easy to think of low income as an example of an environmental stressor, it is also reasonable to think of low perceived control as the perception of existing in a highly stressful environment with which it is difficult to cope-a psychological phenomenon. The link has been demonstrated empirically by many researchers (e.g., Deci \& Ryan, 2002; Lazarus, 1993; Rodin \& Haidt, 1999) in the form of effects of perceptions of control and efficacy on levels of neurotransmitters and endocrine responses. Thus, it seems likely that a gene-environment interaction may underlie the observed phenotypic relationship between physical health and perceived control. Such a mechanism would complement the observed geneenvironment interaction between health and income. Therefore, we hypothesized that the genetic variance associated with physical health would decline with increasing levels of perceived control as well as with increasing income. To the extent that this is true, it reveals an important aspect of the distinction between perception and reality. That is, it suggests that a personality variable involving perception of reality is as powerful as the "actual reality" of income. The purpose of this study was to test this hypothesis.

\section{Method}

\section{Sample}

We used the twin sample described in Johnson and Krueger (2004). In brief, the sample was gathered as part of the MacArthur Foundation National Survey of Midlife Development in the United States (MIDUS; Kessler et al., 2004). The base sample consisted of 998 twin pairs distributed roughly according to population throughout the continental United States and ranging in age from 25 to 74 years. For our study, we used the 719 same-sex pairs for whom we had income, chronic illness, height and weight, control, and zygosity data, resulting in 172 monozygotic (MZ) male pairs, $195 \mathrm{MZ}$ female pairs, 138 dizygotic (DZ) male pairs, and 214 DZ female pairs. Thus, we excluded 262 opposite-sex pairs and 17 pairs with missing or indeterminate zygosity information from the full MIDUS twin sample of 998 pairs. Table 1 shows demographic information for the sample we used for this study.

\section{Measures}

MIDUS participants provided survey data about annual income in several categories, including personal and spousal earnings, Social Security income, and other government assistance that could be used to compute household income. The participants were slightly more wealthy than individuals in the country as a whole, but about $30 \%$ had incomes below the national median, $14 \%$ had incomes below $\$ 20,000$, and $8 \%$ had incomes
Table 1

Sample Demographic Information

\begin{tabular}{|c|c|}
\hline Category & $\%$ \\
\hline \multicolumn{2}{|l|}{ Sex } \\
\hline Male & 43.1 \\
\hline Female & 56.9 \\
\hline \multicolumn{2}{|l|}{ Race } \\
\hline White & 81.2 \\
\hline Black/African American & 3.7 \\
\hline Other & 1.7 \\
\hline Not reported & 13.5 \\
\hline \multicolumn{2}{|l|}{ Age (years) } \\
\hline 25-34 & 23.2 \\
\hline $35-44$ & 28.6 \\
\hline $45-54$ & 26.5 \\
\hline $55-64$ & 13.7 \\
\hline $65-74$ & 7.9 \\
\hline \multicolumn{2}{|l|}{ Education } \\
\hline Under 12 years & 9.8 \\
\hline 12 years & 31.2 \\
\hline Over 12 years & 55.1 \\
\hline Not reported & 3.8 \\
\hline \multicolumn{2}{|l|}{ Annual income } \\
\hline$\$ 0-\$ 10,000$ & 5.1 \\
\hline$\$ 10,001-\$ 20,000$ & 8.6 \\
\hline$\$ 20,001-\$ 35,000$ & 17.2 \\
\hline$\$ 35,001-\$ 50,000$ & 24.5 \\
\hline$\$ 50,001-\$ 75,000$ & 24.3 \\
\hline$\$ 75,001-\$ 100,000$ & 9.1 \\
\hline$\$ 100,000-\$ 150,000$ & 5.9 \\
\hline$\$ 150,000+$ & 5.1 \\
\hline \multicolumn{2}{|l|}{ Marital status } \\
\hline Married & 68.8 \\
\hline Not married & 27.0 \\
\hline Not reported & 4.2 \\
\hline
\end{tabular}

below $\$ 15,000$, cutpoints chosen because they represent limited economic means. To normalize the distribution, we log-transformed the income variable (Cook \& Weisberg, 1999).

The MIDUS surveys also included several questions about physical health. For the present study, we used two measures: number of chronic illnesses and BMI. Participants indicated whether they had been treated for each of 29 chronic health problems in the past 12 months. We summed positive responses to form a total score and log-transformed the variable to make it more normal. The sample was relatively healthy; almost $35 \%$ of participants did not check a single health problem. Over $28 \%$, however, reported treatment for 3 or more of the health problems.

Participants also indicated their current height and weight, which we used to compute their BMIs $\left(\mathrm{m}^{2} / \mathrm{kg}\right)$. Though not necessarily an indicator of current physical health, excessive weight is a well-known risk factor for the development of a number of physical health problems, including diabetes, high blood pressure, heart disease, certain kinds of cancer, and stroke (Komaroff, 1999). Given the problem of obesity in the United States in general and the fact that $56 \%$ of our sample could be considered overweight using the usual criterion of BMI of at least 25 , BMI seemed a simple and relatively objective measure of overall health, even though health risks associated with BMI levels below 25 are not generally agreed upon.

Finally, the MIDUS questionnaire included a series of questions about the degree to which participants perceived they had control over various aspects of their lives, phrased as follows: "Using a 0 to 10 scale, where 0 means 'no control at all' and 10 means 'very much control,' how would you rate the amount of control you have over your ___ these days?" The life aspects queried were health, work situation, financial 
situation, contribution to the welfare and well-being of other people, overall relationship with children, marriage or close relationship, sexual aspect, and life overall. The coefficient alpha for the scale formed from the mean response from these ratings was .73. Participants who did not have spouses, close relationships, or children and thus did not respond to these items received scores based on the means of the items to which they did respond. Of note, the scale reflected control over aspects of life besides health. Item-total correlations for the eight items ranged from .31 to .66; the control over health item ranked fourth highest, with an item-total correlation of .44 . We also repeated the analyses described below using the control scale with the health item omitted. Because results were effectively identical to those based on the scale including the health item, we present only those for the full scale.

\section{Analytical Approach}

The standard quantitative genetic model for a single variable is based on the understanding that the observed (phenotypic) variance $(V p)$ in a trait is a linear function of additive genetic $\left(A^{2}\right)$ variance and shared $\left(C^{2}\right)$ and nonshared $\left(E^{2}\right)$ environmental variance. Additive genetic variance reflects variation in genotypes transmitted from parents to offspring that causes phenotypic variance in the trait. Shared environmental variance (e.g., variation in neighborhoods) reflects variation in environmental characteristics that affects all persons within a family to the same degree and that distinguishes among families. Nonshared environmental variance (e.g., differential educational or occupational experiences) reflects variation in environments that causes individual family members to differ from each other. Error variance is included with nonshared environmental variance because the nonshared environmental variance terms reflect the remaining variance after the effects of additive genetic and shared environmental variance have been estimated. Symbolically,

$$
V \mathrm{p}=A^{2}+C^{2}+E^{2}
$$

Under this model, each of the variance components is independent of the others. To investigate the possibility that genetic variance associated with physical health might decrease with increasing levels of income and/or control, we needed a model in which the variance components themselves vary as continuous functions of the moderators. That is, rather than modeling physical health using the linear equation shown above, we used variance component models for gene-environment interactions described by Purcell (2002) to express the variance associated with each of the three components as a linear interaction with the moderators. For one moderator, this led to the following equation:

$$
\begin{aligned}
V \mathrm{p}=\left(A+\beta_{\mathrm{a}} \times \text { Moderator }\right)^{2}+(C+ & \left.\beta_{\mathrm{c}} \times \text { Moderator }\right)^{2} \\
& +\left(E+\beta_{\mathrm{e}} \times \text { Moderator }^{2} .\right.
\end{aligned}
$$

In this equation, genetic variance $A^{2}$ from Equation 1 is expressed as $\left(A+\beta_{\mathrm{a}} \times \text { moderator }\right)^{2}, C^{2}$ is expressed as $\left(C+\beta_{\mathrm{c}} \times \text { moderator }\right)^{2}$, and $E^{2}$ is expressed as $\left(E+\beta_{\mathrm{e}} \times \text { moderator }\right)^{2}$. The betas are parameter estimates that allow the variance components to differ across moderator levels. Thus, for example, if genetic variance $A^{2}$ at the mean level is .5 and $\beta_{\mathrm{a}}$ is - .1, then people with moderator scores that are 1 standard deviation above the mean would have a genetic variance of $.37\left[(\sqrt{.50}-.1)^{2}\right]$, whereas those with moderator scores 1 standard deviation below the mean would have a genetic variance of .65. In addition, the expected trait level is $\mu+\beta_{\mathrm{m}} \times$ moderator, in which $\mu$ is the overall mean level of health and $\beta_{\mathrm{m}}$ reflects the main effect of the moderator on health. The model is explained in greater detail in Purcell (2002). For the purposes of this study, we extended this model to incorporate both income and perceived control as moderating variables, which meant adding terms for potential interactions among the moderators as well.

Of course, it is possible that genes are involved in the relationship between physical health and either or both moderators through a gene- environment correlation (the genetic control of exposure to different environments), in addition to or instead of through a gene-environment interaction (the genetic control of sensitivity to the environment). If this is the case, it is the result of common genetic influences on both physical health and the environmental moderator. Genetic influences on many variables generally considered to be environmental (including income and control) are well known (Plomin \& Bergeman, 1991). The possibility of a gene-environment correlation can be handled in the model by expressing the moderator means for each individual as linear functions of the overall means, as shown above. This is a natural step, because implementation of the variance model in Equation 2 requires modeling the means as well as the variances. This step effectively removes from the model any genetic effects that are shared between physical health and the environmental moderator. When this is done, such correlated genetic effects will appear as main effects. When both gene-environment correlation and interaction are present, however, this approach may fail to detect gene-environment interactions (Purcell, 2002). That is, any interaction effects detected represent lower bounds on the magnitude of the actual interaction effects in the presence of a gene-environment correlation.

To explore the possible involvement of a gene-environment correlation directly, we had to turn to a different model that could estimate the gene-environment correlation itself (Purcell, 2002). We applied this modified model to estimate the gene-environment correlation between physical health and each moderator (income and control) separately for clarity. In this model, the moderator is used both as a dependent variable to be modeled and as a direct influence on physical health. Thus, the main effect in the means model is replaced with an estimate of genetic effects common to both moderator and physical health, and estimates of genetic variance in physical health from both common and unique sources are provided. The gene-environment correlation thus reflects the degree to which genetic variance is common to physical health and the moderator.

Throughout, we conducted our analyses of twin data using maximumlikelihood estimation for raw age- and sex-corrected (McGue \& Bouchard, 1984) data as operationalized in the computer program Mx (Neale, 1997). This was important, as there were clear effects of both age and sex, particularly in the physical health and income measures. Though the regression process used to remove these effects resulted in standardized data, we also applied our models to unstandardized data and obtained highly similar results; hence, we present only the results based on standardized data. Note that income and BMI reflect real physical scales and thus are not subject to the problems of interval imprecision generally associated with psychological variables (McDonald, 1999). This is somewhat true of number of chronic illnesses as well. We assessed model fit using the likelihood ratio comparison of two likelihood statistics, which is distributed as chi-square, and the Akaike Information Criterion (AIC; Akaike, 1983). AIC is defined as $-2 \times \log$-likelihood for the model, plus two times the number of parameters. Models with smaller AICs are preferred.

Genetic influences can take two basic forms that are treated somewhat differently in quantitative genetic models. Additive genetic influences reflect the independent influence of multiple genes, whereas in our analysis nonadditive genetic influences reflect dominance (alleles that express phenotypic effects even when heterozygous) and other polygenic effects that act like dominance in the sense that they result in DZ twin correlations that are less than half the corresponding MZ twin correlations. Quantitative genetic models are not identified when both additive and nonadditive genetic influences and shared and nonshared environmental influences are modeled at once; one of the parameters must be dropped. In our previous analysis (Johnson \& Krueger, 2004), we had ascertained that number of chronic illnesses showed no evidence of shared environmental influence and did show evidence of nonadditive genetic influences, so we fit models to these data that included parameters reflecting additive and nonadditive genetic and nonshared environmental influences. Because BMI showed no evidence of nonadditive genetic influences but did show some evidence of 
shared environmental influence, we fit models to these data that included parameters reflecting additive genetic and shared and nonshared environmental influences.

Basic correlations among the study variables and between $\mathrm{MZ}$ and DZ twins are shown in Table 2. When the correlations between MZ twin pairs are greater than those between DZ pairs, genetically influenced variance is indicated. When the correlations between MZ pairs are less than twice the correlations between DZ pairs, shared environmentally influenced variance is indicated. Thus, our data suggest that at the overall population level, the systematic variation in income and number of chronic illnesses was primarily under genetic influence. Systematic variation in population-level BMI and control appeared to result from both genetic and shared environmental influences, with genetic influences predominating for BMI and shared environmental influences predominating for control.

\section{Results}

The model-fitting statistics for the gene-environment interaction models we applied are shown in Table 3. For chronic illnesses, we began by asking how the saturated model fit. This first model included estimates of all possible model parameters, additive and nonadditive genetic and nonshared environmental influences and their interactions, as well as main effects for each moderator and their interaction on the mean. We used this model to develop the likelihood ratio test statistics necessary to evaluate the significance of the interaction parameters and moderation effects. We then asked whether the moderation parameters could all be fixed to 0 to test for the existence of any interaction effect. We found that this was not possible without significant loss of model fit, so we went on to estimate each combination of parameters and constraints. As shown in Table 3, fixing the full income interaction (Income $\times \mathrm{A}$, Income $\times \mathrm{C}$, and Income $\times \mathrm{E}$ ) to 0 resulted in increased AIC, as did fixing the full control interaction. However, it was possible to fix the Income $\times$ Control interaction to 0 , though it was not possible to fix either the main effect of income or the main effect of control to 0 . Models with either genetic or nonshared environmental interaction terms were statistically indistinguishable. That is, either term could be fixed to 0 without significant loss of fit, though both could not be so constrained. We thus selected for presentation the model with both terms free, though the resulting AIC was slightly higher than the AIC with either term fixed. We used the same procedure for BMI with very similar results, substituting the estimate of shared environmental influences for nonadditive genetic influence. For BMI, we selected for presentation the model with the lowest AIC.

Table 4 shows the parameter estimates resulting from the selected models, based on the results described above. The table highlights the parameters that were important in the selected

Table 2

Correlations Between Study Variables and Monozygotic (MZ) and Dizygotic (DZ) Twins

\begin{tabular}{lcccccc}
\hline \multicolumn{1}{c}{ Study variable } & 1 & 2 & 3 & 4 & $\mathrm{MZ}$ & $\mathrm{DZ}$ \\
\hline 1. Income & - & & & & .38 & .13 \\
2. Control & .10 & - & & & .33 & .28 \\
3. No. of chronic illnesses & -.16 & -.25 & - & & .42 & .14 \\
4. Body mass index & -.09 & -.16 & .20 & - & .74 & .42 \\
\hline
\end{tabular}

Note. All correlations were significant at $p<.02$. models, and it also shows their relative magnitudes. Thus, for chronic illnesses, the genetic variance parameter at mean levels of both control and income was .832 , and it was reduced by .177 for each standard deviation unit by which individuals' income exceeded the mean and reduced by .048 for each standard deviation unit by which individuals' levels of perceived control exceeded the mean. This result would be squared to obtain the genetic variance associated with a given level of income and perceived control.

There were main effects for both income and control for both physical health measures but no main Income $\times$ Control effects, ${ }^{1}$ nor were there any genetic or environmental interactions with the interaction between income and control. This latter finding acted to simplify the potential model considerably. Both chronic illnesses and BMI showed significant decreases in genetic variance with increasing income, but all environmental influences could be considered fixed across the income dimension. For control, the situation was more complex. Both measures of physical health showed decreasing genetic variance with increasing control, but chronic illnesses also showed decreasing nonshared environmental variance with increasing control, whereas BMI also showed changes in shared environmental variance across the control dimension. The appropriate environmental variance components were modified by the significant environmental interaction parameters in a way directly analogous to the example for genetic variance in chronic illnesses given above; otherwise, they were fixed.

Figures 1 and 2 present graphical descriptions of the relationships implied by these parameter estimates along the dimension of income. The interactive genetic effects of income on both measures of health can be seen clearly in the decreasing slopes of the genetic variance lines on the graphs. The interactive genetic and shared (for BMI) and nonshared (for chronic illnesses) effects of control can be seen in the differences between the lines reflecting the genetic and environmental influences among people of low and high control (1.5 standard deviations above and below the mean). Figures 3 and 4 present the same information along the dimension of control. In this instance, the interactive genetic and environmental effects of control on the health measures can be seen in the decreasing slopes of the variance lines on the graphs, and the interactive genetic effects of income can be seen in the differences between the lines reflecting the genetic and total influences among people of low and high income (1.5 standard deviations above and below the mean). Control did not account for the full interactive

\footnotetext{
${ }^{1}$ Table 3 shows that, using models that decompose variance into components reflecting genetic and environmental influences, we did not detect a phenotypic Income $\times$ Control interaction effect for either chronic illnesses or for body mass index (BMI). This appears to contradict the results of Lachman and Weaver (1998), at least for chronic illnesses, as they did not consider BMI. To investigate this further, we conducted hierarchical linear regressions of our phenotypic variables directly analogous to theirs. For chronic illnesses, the phenotypic Income $\times$ Control interaction term was significant at $p<.001$, replicating their basic results. This suggests that the interaction observable at the phenotypic level can be interpreted more clearly when the variance can be decomposed into its genetic and environmental components. The phenotypic Income $\times$ Control interaction term for BMI was not significant in our data, though those data did reveal a gene-environment interaction process very similar to that for chronic illnesses. We thank an anonymous reviewer for raising this question.
} 
Table 3

Questions and Answers Regarding Relative Fit of Models of Variance Components of Chronic Illnesses and Body Mass Index Moderated by Income and Control

\begin{tabular}{llrrrr}
\hline \multicolumn{1}{c}{ Question } & Answer & LRT & LRT $d f$ & $p$ & AIC \\
\hline Number of chronic illnesses & & & & & \\
$\quad$ How does the saturated model fit? & No & $3,160.4$ & 3,560 & & $3,192.4$ \\
Can all the moderation effects be fixed to 0? & No & 6.7 & 12 & .000 & $3,218.1$ \\
Can the full income interaction be fixed to 0? & No & 6.1 & 3 & .107 & $3,192.5$ \\
Can the full control interaction be fixed to 0? & Yes & 2.2 & 4 & .107 & $3,192.5$ \\
Can the Income $\times$ Control interaction be fixed to 0? & No & 7.0 & 1 & .009 & $3,186.6$ \\
Can the income main effect be fixed to 0? & No & 44.0 & 1 & .000 & $3,197.4$ \\
Can the control main effect be fixed to 0? & & 2.5 & 7 & .927 & $3,180.9$ \\
Selected model (see Table 4 for parameters) & & & & & \\
Body mass index & No & $2,849.9$ & 3,514 & & $2,881.9$ \\
How does the saturated model fit? & No & 22.0 & 3 & .000 & $2,920.1$ \\
Can all the moderation effects be fixed to 0? & No & 22.2 & 3 & .000 & $2,897.9$ \\
Can the full income interaction be fixed to 0? & Yes & 2.9 & 4 & .575 & $2,898.1$ \\
Can the full control interaction be fixed to 0? & No & 5.7 & 1 & .017 & $2,885.8$ \\
Can the Income $\times$ Control interaction be fixed to 0? & No & 3.6 & 1 & .058 & $2,883.5$ \\
Can the income main effect be fixed to 0? & & 8.8 & 7 & .000 & $2,876.7$ \\
Can the control main effect be fixed to 0? & & & & \\
Selected model (see Table 4 for parameters) & & & & \\
\hline
\end{tabular}

Note. $\quad$ LRT $=$ likelihood ratio comparison to the freely estimated (saturated) Genetic $\times$ Environmental Interaction model; AIC = the Akaike Information Criterion. The first entry in the LRT and LRT $d f$ column for each measure of physical health gives the $2 \times \log$-likelihood and degrees of freedom for the freely estimated (saturated) model, including all main and moderation effect parameters.

genetic effects of income on health, nor did income account for the full interactive genetic effects of control on health.

The models explicitly estimating a gene-environment correlation provided some evidence for it, suggesting that common genes contribute to both physical health and to each of the moderating variables. The estimated gene-environment correlations that resulted, however, were not single point values for the relation

Table 4

Estimated Parameters for Selected Gene $\times$ Environment Interaction Models for Chronic Illnesses and Body Mass Index (BMI)

\begin{tabular}{lrr}
\hline \multicolumn{1}{c}{ Parameter } & $\begin{array}{c}\text { Chronic } \\
\text { illnesses }\end{array}$ & BMI \\
\hline Genetic variance & .832 & .809 \\
Shared environmental variance & .000 & -.178 \\
Nonshared environmental variance & .722 & .446 \\
Genetic moderation by income & -.177 & -.153 \\
Shared environmental moderation by income & Fixed & Fixed \\
Nonshared environmental moderation by income & Fixed & Fixed \\
Genetic moderation by control & -.048 & -.125 \\
Shared environmental moderation by control & Fixed & .200 \\
Nonshared environmental moderation by control & -.036 & Fixed \\
Genetic Income $\times$ Control effect & Fixed & Fixed \\
Shared environmental Income $\times$ Control effect & Fixed & Fixed \\
Nonshared environmental Income $\times$ Control effect & Fixed & Fixed \\
Main effect of income & -.110 & -.074 \\
Main effect of control & -.203 & -.057 \\
Main effect of Income $\times$ Control & Fixed & Fixed \\
\hline
\end{tabular}

Note. Variance parameters are squared to estimate actual variance. For chronic illnesses, we estimated nonadditive genetic variance and combined it with additive genetic variance. For BMI, we estimated shared environmental variance. between each combination of variables. In the presence of interactions such as those we detected for both income and control, the extent of gene-environment correlation varies with the levels of the two moderators because the interaction exerts its own effect on the degree of common genetic influence. The gene-environment correlations we calculated on the basis of our parameter estimates for moderator levels at the mean and 1.5 standard deviations above and below the mean are shown in Table 5. As the table shows, overall gene-environment correlations were moderate for income and both measures of health. Overall gene-environment correlations were quite strong for control and both measures of health. Both income and control correlations with chronic illnesses remained relatively stable whether the moderators were low or high, but the income and control correlations with BMI both increased substantially in the presence of high control and decreased in the presence of low control. The strength of the control correlations suggests that the gene-environment interactions we were able to detect involving control may be somewhat understated. Adding complexity to the system of interrelationships among the variables, there were also varying degrees of gene-environment correlation between income and control. The correlation was small for income and control at their means (.09) but very high in high-income environments, though effectively absent in low-income environments. It was moderately negative in high-control environments and moderately positive in low-control environments.

\section{Discussion}

The income-health gradient has long presented a tantalizing puzzle to researchers and policymakers alike. The well-established relationship between sense of control and physical health has helped to explain the gradient, as have the observed phenotypic interactions among physical health, control, and income (Bailis et 


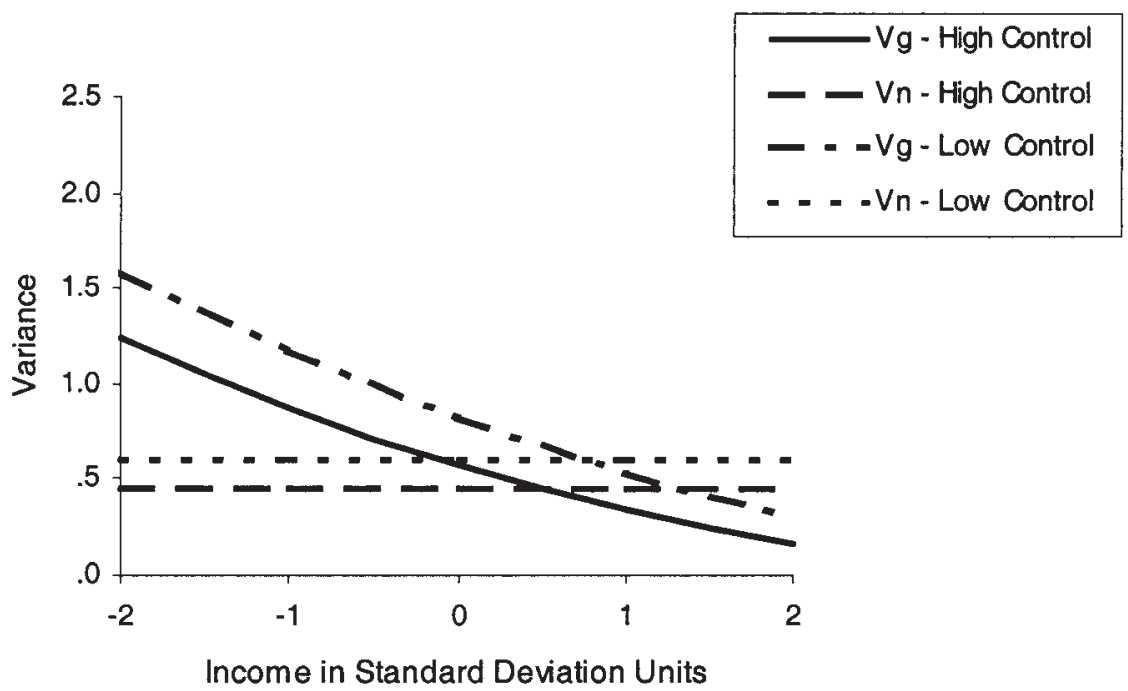

Figure 1. Variance in chronic illnesses as a function of income. $\mathrm{Vg}=$ genetic variance; $\mathrm{Vn}=$ nonshared environmental variance. High and low control are \pm 1.5 standard deviations from the mean for control, respectively. Shared environmental variance was 0

al., 2001; Lachman \& Weaver, 1998), yet the genetic and environmental mechanisms by which income and control might influence physical health have remained unclear. In this study, we supplemented evidence that a gene-environment interaction underlies the income-health gradient by also investigating the extent to which a similar gene-environment interaction underlies the relationship between sense of control and physical health. We found evidence for such an interaction in two measures of physical health, numbers of chronic illnesses and BMI, in a nationwide U.S. twin sample ranging in age from 25 to 74 years. Our results suggest that genetic variance associated with physical health de- creases with increasing income and also with increasing sense of control. The current study should be replicated with larger samples, greater distributions of income and health, and alternative (e.g., physiological) measures of health in order to increase statistical power to quantify effects more precisely. Still, the results present an intriguing picture of the complex interplay between the genetic and environmental influences and the psychological and physical factors involving health.

Gene-environment interaction can be thought of as genetic control of sensitivity to different environmental conditions or, equivalently, as environmental control of different gene effects

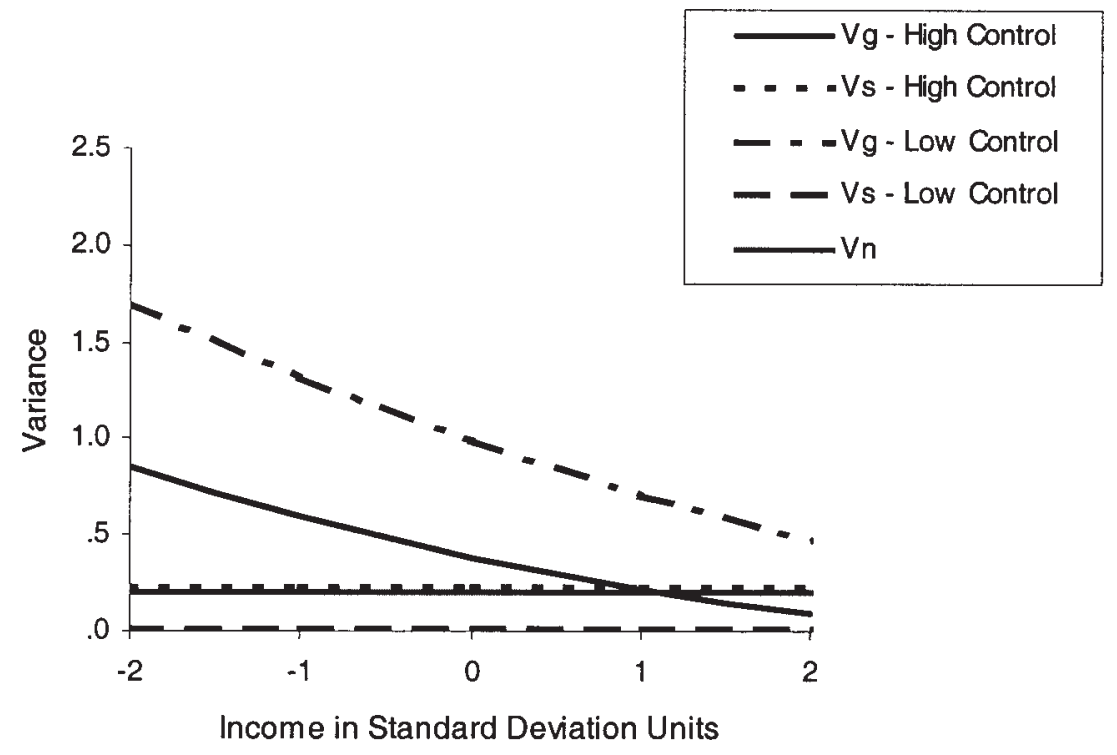

Figure 2. Variance in body mass index as a function of income. $\mathrm{Vg}=$ genetic variance; $\mathrm{Vs}=$ shared environmental variance; $\mathrm{Vn}=$ nonshared environmental variance. High and low control are \pm 1.5 standard deviations from the mean for control, respectively. 


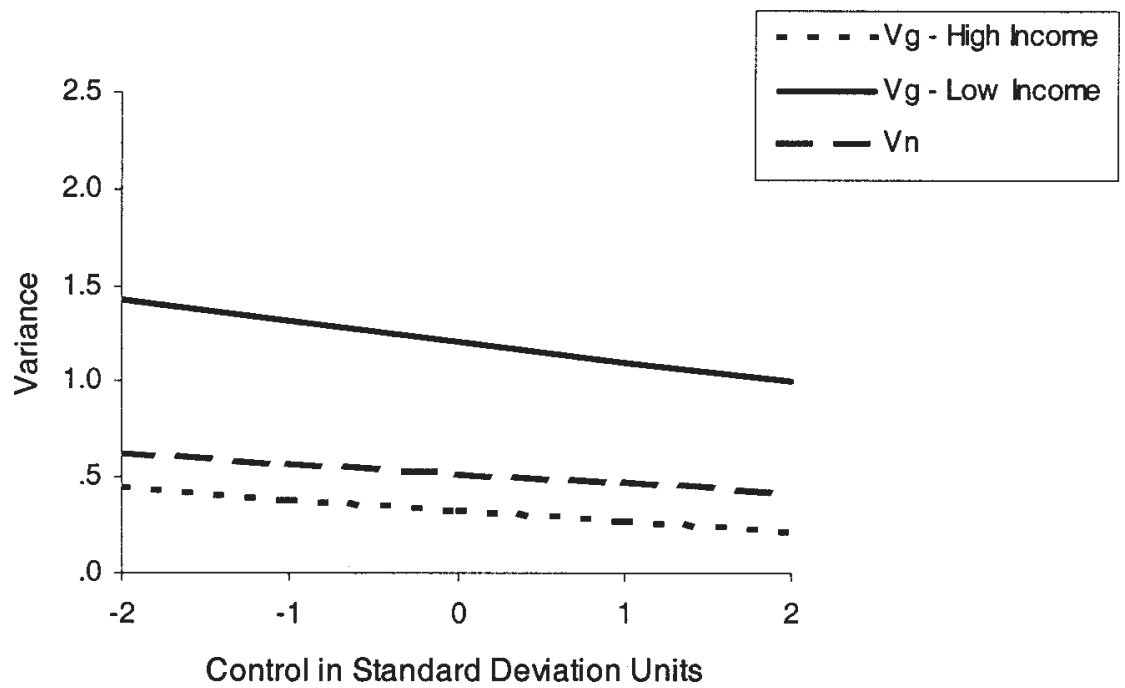

Figure 3. Variance in chronic illnesses as a function of control. $V \mathrm{~g}=$ genetic variance; $V \mathrm{n}=$ nonshared environmental variance. High and low income are \pm 1.5 standard deviations from the mean for income, respectively. Shared environmental variance was 0 .

(Kendler \& Eaves, 1986). It is likely that both conceptualizations have use in thinking about the mechanisms in the differential expression of genetic variance associated with physical health. That is, it seems likely that low-income and/or low-control environments act as objectively observable and/or individually perceived stressors that alter physiological mechanisms such as endocrine responses and neurotransmitter levels, causing them to remain in fight-or-flight mode (Adler, Epel, Castellazzo, \& Ickovic, 2000; McEwen, 1998) and leading, over time (in a manner analogous to the breakdown of a machine running constantly in high gear), to the expression of genetic vulnerabilities in health. Thus, the specific physical health problems that individuals are more likely to encounter arise from their individual genetic heritages, both behavioral and physical. These health effects are reduced in the absence of the stress associated with low-income and/or low-control environments and minimized in the presence of the opportunities associated with higher income and/or the individual's higher level of perceived control. The interpretation of this interaction is complicated by the presence of a geneenvironment correlation.

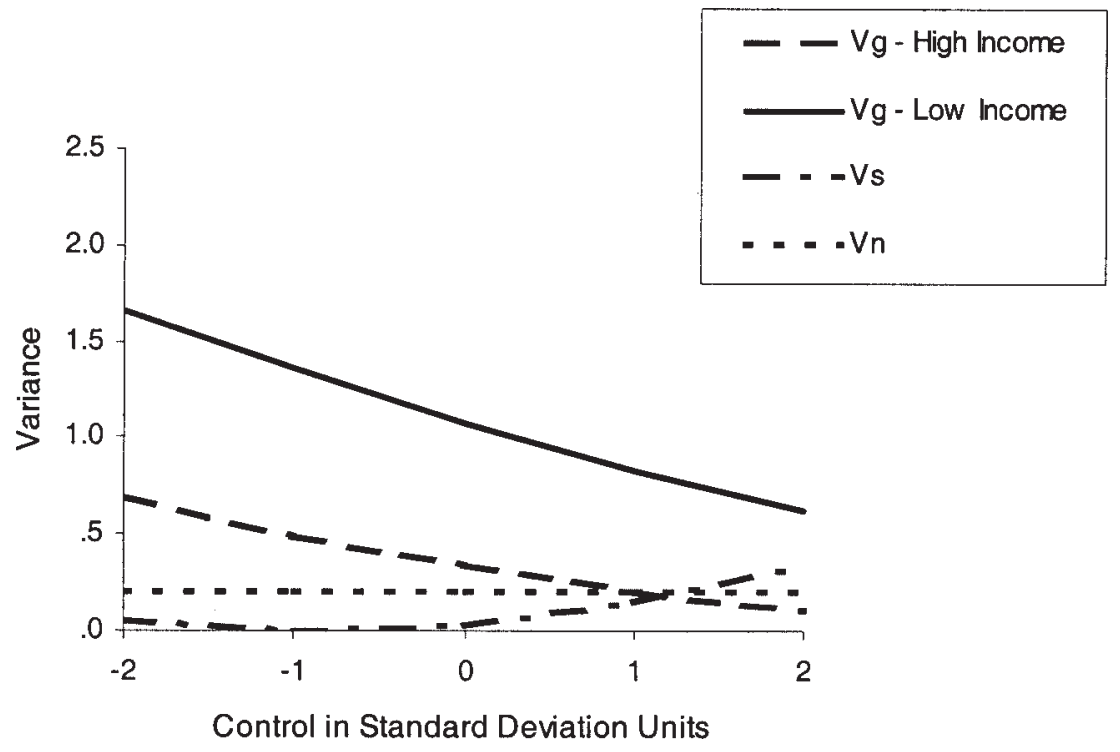

Figure 4. Variance in body mass index as a function of control. $V \mathrm{~g}=$ genetic variance; $V \mathrm{~s}=$ shared environmental variance; $V \mathrm{n}=$ nonshared environmental variance. High and low income are \pm 1.5 standard deviations from the mean for income, respectively. 
Table 5

Gene-Environment Correlations at Mean and at High and Low Levels of Moderators

\begin{tabular}{lccc}
\hline \multicolumn{1}{c}{ Variable } & $M$ & $\begin{array}{c}\text { High } \\
\text { moderator }\end{array}$ & $\begin{array}{c}\text { Low } \\
\text { moderator }\end{array}$ \\
\hline Chronic illnesses-income & -.22 & -.27 & -.20 \\
BMI-income & -.31 & -.57 & -.14 \\
Chronic illnesses-control & -.66 & -.62 & -.69 \\
BMI-control & -.71 & -.93 & -.47 \\
Income & .09 & -.30 & .33 \\
Control & .09 & .88 & -.01 \\
\hline
\end{tabular}

Note. High values of the moderators were defined as 1.5 standard deviations above the mean, low values as 1.5 standard deviations below the mean. For income-health correlations, the moderator is income. For control-health correlations, the moderator is control. For the correlation between income and control, the moderator is first control, then income. $\mathrm{BMI}=$ body mass index.

There was evidence for a gene-environment correlation among the variables we considered, particularly for the measures of physical health and control, and these correlations tended to be higher in high-income, high-control environments (except for the correlation between chronic illnesses and control, which was relatively stable). These correlations would have acted to understate the estimates of the extent of moderation of genetic influences on physical health by the phenotypes of income and control, but they also specify another way in which the income and control are interrelated with physical health. For example, referring to the high correlations between physical health and control, it would appear that people genetically disposed to perceive that they have control over their lives seek out situations in which they can exert such control, and their success in doing so acts to minimize potential deleterious genetic effects on their physical health. To a lesser but still important extent, people who are genetically disposed to be in a position to earn a high income also seek out such situations, and their success in doing so has the same result. It seems likely that the very high gene-environment correlation (.88) between income and control in high-income environments reflects the tendency of similar (e.g., personality and ability) traits to affect both an individual's perception of control and income level. At the same time, it seems possible that an individual's perception of control may reflect learned experience with attempts to exert control, which may explain the lower gene-environment correlations in lowincome and low-control environments. This potentially sheds considerable light on some of the mechanisms underlying the incomehealth gradient and generates possibilities for future research that we intend to pursue. However, these are correlations, and the usual cautions about causal relationships between correlated variables apply. We can only speculate at present about the direction and extent of selection involved in these associations.

The incidence and progression of adult-onset diabetes can be used as a convenient model for thinking about the mechanisms involved. The disease is a debilitating condition whose incidence should be reflected in both of the measures of physical health we used in this study. It is generally accepted that some people have a genetic predisposition for this form of diabetes. It is also well known that the progression of the disease can be minimized, and development of the condition can sometimes even be prevented, through environmental interventions such as dieting, exercising, and monitoring blood sugar levels, all of which obviously involve exertion of personal control. It is also likely that these personal control activities are easier to carry out in a high-income environment, in which education levels tend to be higher, a wider variety of appropriate foods can be made readily available, and gym fees or sports equipment costs do not present an undue burden.

The gene-environment correlations, particularly those for control, can be thought of in similarly concrete terms. The correlations suggest that a common set of genes contributes both to greater physical health and to higher income and that another, possibly different, common set of genes contributes both to greater physical health and to greater control, and these relationships are generally stronger in high-income environments than in low-income environments. These relationships could result directly from the experience of greater physical health: People in good health are less likely to miss work or school because of health problems and are more prepared to carry out their jobs with energy and competence, which may lead over the long run to higher income. Similarly, people in good health tend to have the personal resources to exert control over their lives, which may lead to positive experiences associated with having exerted control and, therefore, to greater perceived ability to exert control. All of this may take place more readily in high-income environments than in low-income environments because of the greater resources and fewer constraints associated with the high-income environment. The geneenvironment correlation for income and control suggests that a common set of genes contributes both to higher income and higher control among people in relatively high-income environments but not in low-income environments.

The existence of gene-environment interactions has long been suspected and has drawn increasing attention as a possible mechanism explaining phenotypic associations. Recently, for example, Turkheimer, Haley, Waldron, D’Onofrio, and Gottesman (2003) demonstrated that variance in IQ could be attributed primarily to shared environmental influences in children of low socioeconomic status. Genetic influences in these children were largely absent. The reverse was true of children of high economic status: The influences accounting for variance in IQ were primarily genetic in these children. This is directly analogous to the effect we observed on physical health, but in the opposite direction in the sense that, for physical health, genetic variance was compressed in more favorable environments, whereas for IQ, genetic variance was compressed in less favorable environments. We speculate that the direction of effect is related to the adaptiveness of the trait in question. High IQ is an adaptive trait, and the more favorable environment encourages its genetic expression. Genetic vulnerabilities to physical illness are not adaptive traits, and the more favorable environments limit their expression.

Gene-environment interactions, whether between IQ and income or health and income, suggest that the objective environment moderates gene expression. This is an important observation and raises interesting possibilities for public policy. But the geneenvironment interaction between health and control is potentially of even greater significance, because control is a psychological phenomenon. That is, the existence of the interaction between health and control suggests that the subjective environment (one's perceptions of one's circumstances) can have effects on gene expression that are as significant as those of objective environ- 
mental circumstances. This raises fascinating possibilities. For example, could psychological interventions that enhance people's perceptions of control over their lives be as effective as public health policies intended to moderate expression of genetic vulnerability to illness? Our results suggest that family members who share low-income environments also tend to share health problems, something that has been observed in many geographic and historical contexts. Although reduction of poverty and the life stresses often associated with it may help to reduce those shared health problems, it may be possible to achieve similar public health results through policies intended to help people attain control over their lives and manage their genetic vulnerabilities to health problems.

Behavioral genetic studies have been criticized for producing static heritability statistics that provide little insight about what are clearly dynamic processes. Such criticisms have merit, but behavioral genetic methodology continues to evolve. Recent advances in methodology such as those applied in this study make clear that the basic behavioral genetic approach can be used to capture dynamic interplay among variables in a way that sheds light on the underlying processes.

\section{References}

Adler, N. E., Boyce, T., Chesney, M. A., Cohen, S., Folkman, S., Kahn, R. L., \& Syme, S. L. (1994). Socioeconomic status and health. American Psychologist, 49, 15-24.

Adler, N. E., Epel, E. S., Castellazzo, G., \& Ickovic, J. R. (2000). Relationship of subjective social and objective social status with psychological and physiological functioning: Preliminary data in healthy White women. Health Psychology, 79, 586-592.

Adler, N. E., \& Snibbe, A. C. (2003). The role of psychosocial processes in explaining the gradient between socioeconomic status and health. Psychological Science, 12, 119-123.

Akaike, H. (1983). Information measures and model selection. Bulletin of the International Statistical Institute, 50, 277-290.

Bailis, D. S., Segall, A., Mahon, M. J., Chipperfield, J. G., \& Dunn, E. M. (2001). Perceived control in relation to socioeconomic and behavioral resources for health. Social Science and Medicine, 52, 1661-1676.

Cook, R. D., \& Weisberg, S. (1999). Applied regression including computing and graphics. New York: Wiley.

Deci, E. L., \& Ryan, R. M. (2002). Handbook of self-determination research. Rochester, NY: University of Rochester Press.

Johnson, W., \& Krueger, R. F. (2004). Genetic effects on physical health: Lower at higher income levels. Manuscript submitted for publication.
Kendler, K. S., \& Eaves, L. (1986). Models for the joint effect of genotype and environment on liability to psychiatric illness. American Journal of Psychiatry, 143, 279-289.

Kessler, R. C., Gilman, S. E., Thornton, L. M., \& Kendler, K. S. (2004). Health, wellbeing, and social responsibility in the MIDUS twin and sibling subsamples. In O. G. Brim, C. D. Ryff, \& R. C. Kessler (Eds.), How healthy are we? A national study of wellbeing at midlife (pp. 124-152). Chicago: University of Chicago Press.

Komaroff, A. L. (Ed.). (1999). Harvard Medical School family medical guide. New York: Simon \& Schuster.

Lachman, M. E., \& Weaver, S. L. (1998). The sense of control as a moderator of social class differences in health and well-being. Journal of Personality and Social Psychology, 74, 763-773.

Lazarus, R. S. (1993). From psychological stress to the emotions: A history of changing outlooks. Annual Review of Psychology, 44, 1-21.

McDonald, R. P. (1999). Test theory: A unified treatment. Mahwah, NJ: Erlbaum.

McEwen, B. S. (1998). Protective and damaging effects of stress mediators. New England Journal of Medicine, 338, 171-179.

McGue, M., \& Bouchard, T. J., Jr. (1984). Adjustment of twin data for the effects of age and sex. Behavior Genetics, 14, 325-343.

Neale, M. C. (1997). Mx: Statistical modeling. Richmond: Medical College of Virginia, Department of Psychiatry.

Parsons, P. A. (2002). Aging: The fitness-stress continuum and genetic variability. Experimental Aging Research, 28, 347-359.

Parsons, P. A. (2003). From the stress theory of aging to energetic and evolutionary expectations for longevity. Biogerontology, 4, 63-73.

Plomin, R., \& Bergeman, C. S. (1991). The nature of nurture: Genetic influences on "environmental" variables. Behavioral and Brain Sciences, 14, 373-427.

Purcell, S. (2002). Variance component models for gene-environment interaction in twin analysis. Twin Research, 5, 554-571.

Rodin, J. (1986, August 14). Aging and health: Effects of the sense of control. Science, 233, 1271-1276.

Rodin, J., \& Haidt, J. (1999). Control and efficacy as interdisciplinary bridges. Review of General Psychology, 3, 317-337.

Rodin, J., Timko, C., \& Harris, S. (1985). The construct of control: Biological and psychosocial correlates. In C. Eisdorfer, M. P. Lawton, \& G. L. Maddox (Eds.), Annual review of gerontology and geriatrics (pp. 3-55). New York: Springer.

Turkheimer, E., Haley, A., Waldron, M., D’Onofrio, B., \& Gottesman, I. I. (2003). Socioeconomic status moderates heritability of IQ in young children. Psychological Science, 14, 623-628.

Received January 21, 2004

Revision received July 21, 2004

Accepted July 23, 2004 